
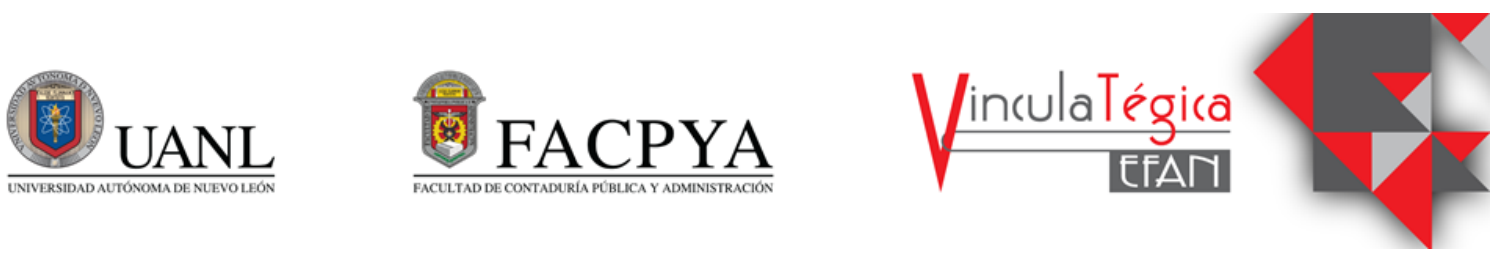

\title{
El clima laboral: todo un reto en tiempo de crisis
}

\author{
María Eugenia Reyes Pedraza ${ }^{1}$, María Delia Téllez Castilla ${ }^{2}$ y Janet García González ${ }^{3}$ \\ ${ }^{1}$ Universidad Autónoma de Nuevo León, Facultad de Contaduría Pública y Administración, México, \\ kenna.reyes@gmail.com Av.Universidad S/N C.P.66451 Col.Ciudad Universitaria, San Nicolás de los \\ Garza Nuevo León, México. Tel. 8116006179. \\ ${ }^{2}$ Universidad Cuauhtémoc, Educación a Distancia Plantel Aguascalientes, México, \\ tellezdelia@yahoo.com.mx Adolfo López Mateos No. 100, CP 20116 Col. Trojes de Alonso Aguascalientes, \\ Aguascalientes, México. Tel. 8182872818. \\ ${ }^{3}$ Universidad Autónoma de Nuevo León, Facultad de Ciencias de la Comunicación, México, \\ janetgarcia71@yahoo.com.mx Paseo del Acueducto, Liverpool S/N Col. Del Paseo Residencial, C.P. 64920, \\ Monterrey Nuevo León, México. Tel. 8112759309.
}

Información del artículo revisado por pares

Fecha de aceptación: junio-2021

Fecha de publicación en línea: diciembre-2021

DOI: https://doi.org/10.29105/vtga7.2-68

\section{RESUMEN}

El clima laboral también se gestiona, los directivos de la organización son los encargados de realizar esta tarea fundamental para una vida laboral saludable. El objetivo de esta investigación fue analizar el Clima Laboral del área de Recursos Humanos del Organismo dedicado a proporcionar los Servicios de Salud en el Estado de Nuevo León. Metodología: Se realizó una investigación descriptiva de corte transversal, de diseño no experimental, los datos primarios fueron obtenidos mediante la técnica de la encuesta. Resultados: Se encontró que no todos los empleados perciben el clima laboral como sano y armonioso, pero el 98\% de ellos comenta estar comprometido con la Organización. Conclusiones: el Organismo debe mantener los rubros donde se le reconocen fortalezas como el sentido de pertenencia y el compromiso de los empleados de contribuir para el logro de los resultados organizacionales y seguir trabajando para mejorar otros rubros como estimular las actividades de convivencia.

Palabras clave: clima laboral, liderazgo, política pública.

\section{ABSTRACT}

The work environment is also managed; the organization's managers are in charge of this task, which is fundamental to keep a healthy work environment. The objective of this research was to analyze the Work Environment in the Human Resources Area of the Organization dedicated to provide health services in the State of Nuevo León. Methodology: a non-experimental descriptive cross-sectional research has been made; primary data has been obtained through the survey technique. Results: It was found that not all employees perceive their work environment as healthy and harmonious, but $98 \%$ of them say they are committed to the organization. Conclusions: The Agency should maintain the areas where strengths are recognized, such as the sense of belonging and the employees' commitment to contribute to the achievement of organizational results, and continue working to improve other areas, such as stimulating coexistence activities.

Keywords: work environment, leadership, public policy.

\section{1.- INTRODUCCIÓN}

El abrupto cambio en la forma de trabajar a partir del año 2020 derivado de la pandemia, hace indispensable analizar el clima laboral 
(CL) en especial en los lugares donde sí se siguió laborando de forma presencial, como es el caso del área de recursos humanos de los Servicios de Salud en el estado de Nuevo León.

Ya lo dijo Ospino (2019) "No siempre la interacción con los pares y compañeros de trabajo son ideales. Cotidianamente surgen conflictos y las decisiones tomadas afectan positiva o negativamente la relación con los integrantes e influyen en la productividad, la calidad del trabajo y el trato hacia los usuarios".

Y continúa explicando que "Las habilidades socioemocionales requieren de formación y práctica generalizada para lograr resultados en la productividad de los trabajadores, pero resolver conflictos depende mucho del tipo de liderazgo que se ejerza; de ahí la importancia de un buen liderazgo ya que existirá repercusión en el CL".

Cardona (2020) menciona fehacientemente que "El ser humano es un ser social. Requiere de la interacción con los demás y lograr objetivos. Surge así la necesidad de trabajar en equipo en las organizaciones; Aparecen sentimientos positivos y negativos que al manejarse adecuadamente no afectan los resultados buscados".

"En un equipo de trabajo dice Cardona (2020) las personas tienen objetivos específicos, trabajan coordinadamente y la carga de trabajo no es necesariamente equitativa. Esto genera el CL dentro de una organización donde las relaciones interpersonales y el liderazgo en los equipos juegan un rol preponderante". Por lo que es importante cuidar del desarrollo de estas habilidades.

Los comportamientos, el modo de trabajar y de relacionarse entre los mismos empleados, la forma de como interactúan con la jerarquía y superiores, los materiales, los equipos, la tecnología y la misma actividad de cada integrante de la organización, son elementos fundamentales para que inicie, crezca y se desarrolle un clima organizacional sano o áspero. Es decir, la parte humana y física de las personas alimentan la cotidianidad, sin olvidar que el estilo de liderazgo es totalmente influyente en este proceso.

Pues resulta entonces que el CL es consecuencia de las percepciones de todos los involucrados en el proceso desde lo más alto de la jerarquía hasta el último nivel de la organización, lo que incluye conocimientos, habilidades, experiencias, actividades, interacciones y valores de cada uno de ellos; que a su vez se combinan con las estructuras, funciones, procesos metas, objetivos y valores organizacionales lo que hace formen entre todos un mismo sistema que por supuesto impacta en los resultados obtenidos, que hablando de los servicios de salud se convierte en una atención de calidad. Gómez (2004) explica que "No existe un listado único de las características que definen el CL, aunque hay consenso acerca de su carácter multidimensional". Por lo anterior, al área de Recursos Humanos de las Organizaciones le toca provocar, indicar, coadyuvar y dar seguimiento al tema de CL en aras de mantener el equilibrio laboral.

Analizando esta situación, surge la pregunta de investigación. ¿Cuál es el estado actual del Clima Laboral en el Área de Recursos Humanos del Organismo dedicado a la Salud en el Estado de Nuevo León? y de ella deriva el Objetivo de esta investigación que es: analizar el Clima Laboral del Área de Recursos Humanos del Organismo dedicado a proporcionar los servicios de Salud en el Estado de Nuevo León.

\section{2.- MARCO TEÓRICO \\ 2.1 Clima Laboral}

Hoy día los trabajadores de la salud forman parte de una historia inimaginable en el andar de la vida laboral. El afrontar un terrible padecimiento desconocido al que había que hacer frente, los conocimientos, las habilidades, las actitudes, el liderazgo y la comunicación son habilidades gerenciales indiscutibles para el fortalecimiento del clima laboral en las organizaciones de salud.

Hablar de clima laboral, es hablar de la influencia de múltiples factores. Chiavenato (2011) afirma que "Cada organización tiene su cultura organizacional o corporativa. Para conocer una organización, el primer paso es 
comprender esta cultura... Formar parte de una organización significa asimilar su cultura... representa las normas informales, no escritas, que orientan el comportamiento cotidiano" (p. 72). Son estas normas no escritas, estos componentes invisibles como por ejemplo los sentimientos grupales, las actitudes, las expectativas, los afectos de cada integrante del equipo, lo que construye el clima laboral, que es lo que los individuos perciben.

Schein (1992) definió Cultura Organizacional como "El conjunto de hábitos y creencias establecido por medio de normas, valores, actitudes y expectativas que comparten todos los miembros de la organización y que refleja la mentalidad predominante".

Para Schein (1992) "Es un patrón de aspectos básicos compartidos inventados, descubiertos o desarrollados por un grupo que aprende a enfrentar sus problemas de adaptación externa e integración interna, deseable su transmisión a nuevos miembros, como forma correcta de percibir, pensar y sentir".

Chiavenato (2017) continúa diciendo que "La comparación con un iceberg tiene una razón evidente: la cultura organizacional presenta estratos de profundidad y arraigo y para conocer la cultura de una organización es necesario observar todos esos niveles. Mayor profundidad de arraigo mayor dificultad de cambio".

Luthans, Fred (2002) comento que "La cultura organizacional refleja la manera como cada organización aprendió a manejar su ambiente. Es una mezcla compleja de prejuicios, creencias, comportamientos, historias, mitos, metáforas y otras ideas que, juntas, representan el modo particular de trabajar y funcionar de una organización".

Luthans, Fred (2002) continua afirmando que "La cultura organizacional presenta seis características principales: regularidad en los comportamientos observados; normas; valores predominantes; filosofía; reglas y; clima organizacional".

"La definición de clima organizacional es compleja, una gama que enmarca dos puntos de vista extremos: los que enfatizan en factores organizacionales puramente objetivos, y los que privilegian los atributos percibidos por los individuos dentro de la organización". (Noriega, 2015)

"La especial importancia de este enfoque reside en el hecho de que el comportamiento de un trabajador no es una resultante de los factores organizacionales existentes, sino que depende de las percepciones que tenga el trabajador de estos factores". (Noriega, 2015)

"Sin embargo, estas percepciones dependen en buena medida de las actividades, interacciones y otra serie de experiencias que cada miembro tenga con la organización. De ahí que el clima organizacional refleje la interacción entre características personales y organizacionales". (Noriega, 2015)

Chiavenato (2017) expone que "Como consecuencia del concepto de motivación (en el nivel individual) surge el concepto de clima organizacional (en el nivel de la organizacional) como aspecto importante de la relación entre personas y organizaciones". (P. 74).

Chiavenato (2017) continúa expresando que "Las personas están en continua adaptación para satisfacer sus necesidades y mantener equilibrio individual. Esta adaptación no se restringe a la solución de necesidades fisiológicas y de seguridad, también comprende la satisfacción de las necesidades sociales de estima y autorrealización”. (P. 74).

Chiavenato (2017) y que "Como la satisfacción de las necesidades superiores depende mucho de otras personas, en especial de las que ocupan posiciones de autoridad, es importante comprender la naturaleza de la adaptación; varía según la persona y el momento en que se encuentre". (P. 74).

Chiavenato (2017) de acuerdo a lo anterior indica que "las tres características principales de las personas mentalmente sanas son: las personas se sienten bien consigo mismas; se sienten bien en relación con otras personas y; son capaces de enfrentar las demandas de la vida y de las circunstancias". 
Además, Chiavenato (2017) explica que "El clima organizacional está íntimamente relacionado con la motivación de los miembros de la organización. Cuando la motivación entre los participantes es elevada, el clima organizacional tiende a ser elevado, proporcionando relaciones de satisfacción, ánimo, interés y colaboración entre los participantes". (P. 74).

Por lo tanto, Chiavenato (2017) termina diciendo que "El concepto de clima organizacional expresa la influencia del ambiente sobre la motivación de los participantes, que se puede describir como la cualidad $o$ propiedad del ambiente organizacional que perciben o experimentan sus miembros y que influye en su conducta". (P. 74).

"Las relaciones de los empleados con la organización contemplan las generadas por la estructura formal y las interpersonales e intergrupales. Los patrones de comportamiento de las personas son resultado, no solo de los valores, normas y pautas propias de la estructura organizacional, sino también de las condiciones que se establecen en las interacciones. La cultura organizacional incide en el vínculo entre la estructura y el individuo dentro de la empresa". (Prieto, 2017, p. 70). Stephen Robbins E. (1996) dice que "El concepto de clima organizacional se puede asimilar al de cultura organizacional. En ese orden de ideas, explica el concepto más usado para describir la personalidad de una empresa es el clima, es preferible utilizar el de cultura".

Stephen Robbins E. (1996) continúa diciendo que "No solo para enfatizar que las organizaciones tienen diferentes grados de calor humano, sino también para reafirmar que cuenta con tradiciones, valores, costumbres, prácticas y procesos de socialización que prevalecen durante el tiempo".

Brunet (1983) definió la importancia del clima organizacional como "Una forma global que refleja los valores, las actitudes y las creencias de los miembros, que debido a su naturaleza, se trasforman a su vez en elementos del clima. Así se vuelve importante para un administrador el ser capaz de analizarlo y diagnosticarlo". (p. 20).

Brunet (1983) También dijo que "El clima organizacional se diagnostica por tres razones: evaluar las fuentes de conflicto, de estrés o de insatisfacción; iniciar y sostener un cambio; seguir el desarrollo de su organización y prever los problemas que puedan surgir". (p. 20).

Por lo tanto, dice Brunet (1983) que "El administrador puede ejercer un control sobre la determinación del clima laboral de manera tal que pueda administrar lo más eficazmente posible su organización". (p. 20).

El análisis de clima organizacional en sus diversas versiones debe contener aspectos psicosociales tales como Prieto (2017) lo concluye:

Conocimiento de los objetivos, compromiso hacia las metas, conformidad frente al liderazgo institucional, motivación y satisfacción en el trabajo, calidad en las relaciones interpersonales, participación en la toma de decisiones, flujo efectivo de la comunicación, amor al trabajo con significado, organización del trabajo, recompensa del trabajo, apoyo a la supervisión funcional, ayuda a los compañeros, trabajo en equipo, sinergia organizacional (p. 74)

Según Chiavenato (2009) "el liderazgo es una combinación de tres fuerzas: la del líder propiamente, la de los subordinados y las fuerzas de la situación. Esta interacción es la que brinda las pautas para que se defina el estilo de liderazgo que se pueda implementar".

\subsection{Política pública}

El empleo es un factor esencial que permite la integración y cohesión social, al mismo tiempo que brinda una identidad al individuo dentro una sociedad y la política pública laboral es la que vincula la acción política, las leyes y el empleo con el enfoque que el gobierno considere (Sánchez Torres, 2011). Si bien en México los derechos laborales están consignados en la constitución mexicana desde el siglo pasado, no necesariamente están actualizados o se cumplen puntualmente.

En el rubro de política pública de salud, particularmente en la salud mental de los empleados, se han realizado protocolos de 
actuación frente a la violencia laboral y el hostigamiento, para que los empleados tengan un ambiente laboral sano y además se respete su dignidad. La expresión moobing se utiliza en casos de persecución psicológica y de acoso laboral (Lugo Garfias, 2017).

La Organización Internacional del Trabajo (OIT) y la Organización Mundial de la Salud (OMS) se unen en la disciplina de medicina del trabajo para fomentar la salud de los trabajadores, su productividad y la relación de estas con el medio ambiente generando un clima de salud y seguridad laboral (Álvarez Torres y Riaño-Casallas, 2018). Todo esto con la intención de lograr un bienestar para cada empleado y con ello lograr mayor productividad y además el desarrollo sostenible que se pretende hoy día.

\section{3.- MÉTODO}

Se realizó una investigación descriptiva de corte transversal, de diseño no experimental. Con datos primarios obtenidos mediante la técnica de la encuesta. En una muestra por conveniencia donde se encuestaron a 54 personas, conformada por el grupo de colaboradores del área de Recursos Humanos del Organismo dedicado a la Salud del Gobierno del Estado de Nuevo León. La recolección de los datos se obtuvo en abril de 2021. Los participantes fueron contactados en su lugar de trabajo y dentro de su horario laboral. Como criterio de inclusión se consideró a todos los empleados del área contratados al momento de la aplicación y con interés de participar en el estudio.

Se explicó el objetivo del estudio y se garantizó la confidencialidad y el anonimato por lo que no se solicitó su nombre. La encuesta fue enviada a su dispositivo móvil y contestada en la privacidad de su lugar de trabajo. El cuestionario digital permitió recolectar el $100 \%$ de las respuestas, ya que era requisito que la encuesta estuviera completa para poder ser enviada una vez concluida, lo que admitió que todas las encuestas fueran contabilizadas.

El cuestionario sobre Clima Laboral fue de elaboración propia para conocer el grado de satisfacción con el mismo por parte de los empleados del área.
Para recolectar los datos se utilizó como herramienta un formulario digital con respuestas cerradas en escala de Likert. Las respuestas tipo Likert contuvieron cinco categorías: totalmente de acuerdo, de acuerdo, ni de acuerdo ni en desacuerdo, en desacuerdo y totalmente en desacuerdo. Para el análisis estadístico se utilizó estadística descriptiva, para observar la distribución de los resultados.

Como no existe evaluación previa de esta índole, se desconoce la prevalencia de este problema.

\section{4.- RESULTADOS}

El perfil demográfico de los encuestados muestra que la edad de los mismos fluctúa entre menos de 30 años y más de 60 años cronológicos; el $20 \%$ son hombres, el $67 \%$ mujeres y el $13 \%$ decidió no decirlo; la escolaridad muestra que el $13 \%$ tiene nivel de posgrado, el $59 \%$ carrera profesional, $11 \%$ carrera técnica, el $15 \%$ preparatoria y solo un $2 \%$ cuenta con nivel de secundaria.

En cuanto al perfil laboral, de la totalidad de encuestados el $13 \%$ cuenta con un nombramiento de subdirector o jefe de departamento, $24 \%$ tiene puesto de coordinador y el 63\% ocupa puestos operativos de acuerdo al departamento asignado. El mayor porcentaje, 37\% tiene una antigüedad de 6 a 10 años, $17 \%$ menor de 5 años, el $22 \%$ de 11 a 20 años, el $13 \%$ de 20 a 30 años y el $11 \%$ tiene más de 30 años trabajando para el Organismo; puede ser que los años trabajados hayan sido en diferentes áreas del Organismo.

En la figura 1 se representan las respuestas al ítem "Conozco los objetivos de la SSNL", obteniéndose el $72 \%$ de totalmente de acuerdo y de acuerdo, el $28 \%$ ocupa los rubros de ni de acuerdo ni en desacuerdo, en desacuerdo y totalmente en desacuerdo. 


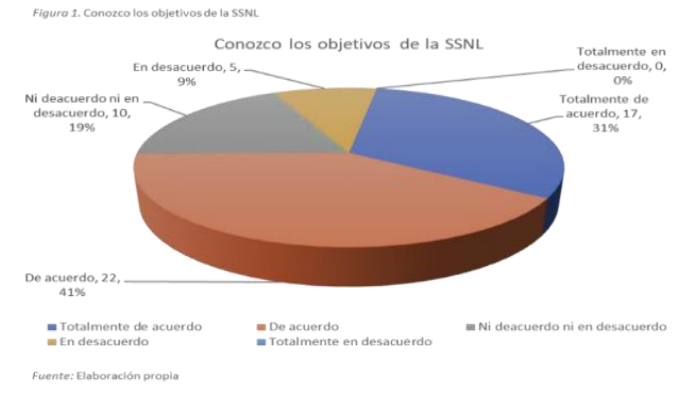

Respecto al ítem: "Mi director explica los motivos que hay detrás de las decisiones y acciones tomadas", el 24\% expresó estar de totalmente de acuerdo, el 39\% dijo estar de acuerdo, 26\% ni de acuerdo ni en desacuerdo, $2 \%$ en desacuerdo y el $9 \%$ totalmente en desacuerdo.

Los resultados en relación a "Mi director me inspira a desempeñar mi trabajo de la mejor manera" el 30\% dijo estar totalmente de acuerdo, $41 \%$ de acuerdo, $17 \%$ ni de acuerdo ni en desacuerdo, $7 \%$ en desacuerdo y el 5\% totalmente en desacuerdo. En los primeros dos rubros se suma más del $70 \%$.

En la figura 2 se observan las respuestas a la pregunta "Me siento motivado por el trabajo que desempeño", el $42 \%$ dijo estar totalmente de acuerdo y el $37 \%$ está de acuerdo, la suma del resto de los rubros solo asciende a un $21 \%$ expresan estar totalmente en desacuerdo, en desacuerdo y ni de acuerdo ni en desacuerdo.

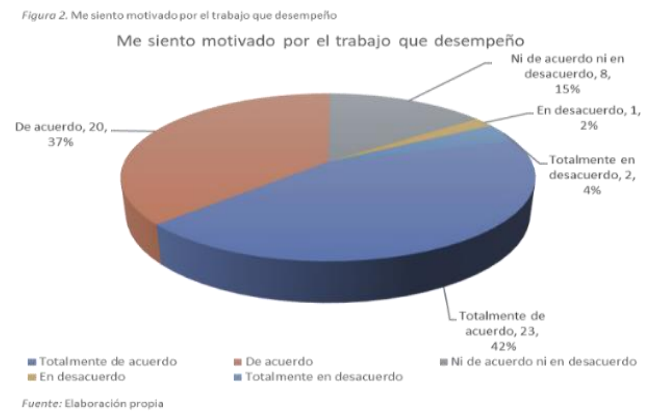

Para el cuestionamiento de si "Mi trabajo me ocasiona mucho estrés" los datos arrojados están en la figura 3 y fueron que solo un $4 \%$ se encuentra totalmente de acuerdo, $6 \%$ de acuerdo, $35 \%$ ni de acuerdo ni en desacuerdo, $35 \%$ en desacuerdo y el $20 \%$ totalmente en desacuerdo.

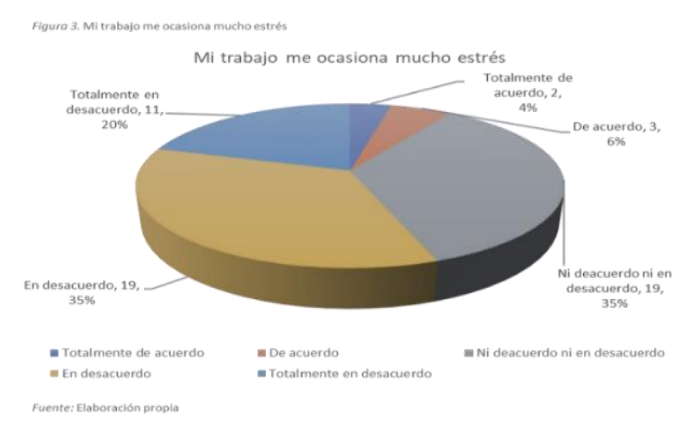

Para la pregunta "Sería más feliz en otra dependencia" el resultado es que $0 \%$ dice estar totalmente de acuerdo, $4 \%$ de acuerdo, $31 \%$ ni de acuerdo ni en desacuerdo, $39 \%$ en desacuerdo y el $26 \%$ totalmente en desacuerdo.

La figura 4 contiene las respuestas de si "Los lideres (oficinas centrales) tratan de mantener el orden y colabora en el equipo con el personal" aquí el 18\% dijo estar totalmente de acuerdo, $57 \%$ de acuerdo, $17 \%$ ni de acuerdo ni en desacuerdo, $6 \%$ en desacuerdo y el $2 \%$ totalmente en desacuerdo.

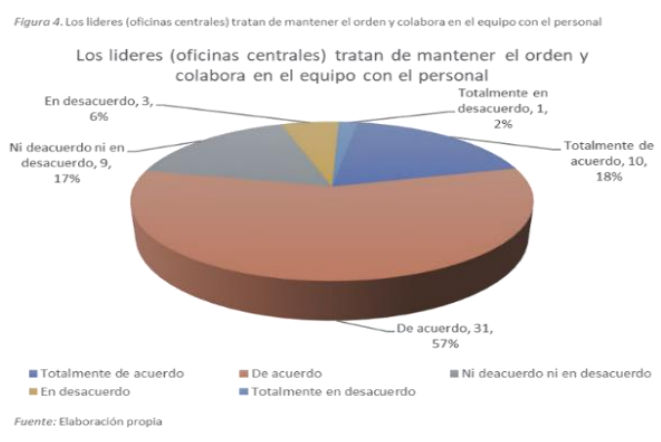

Para el cuestionamiento de "Tengo el compromiso de seguir mejorando el trabajo en equipo" el $57 \%$ dijo estar totalmente de acuerdo, 39\% de acuerdo, $4 \%$ ni de acuerdo ni en desacuerdo, $0 \%$ en los rubros de desacuerdo y totalmente en desacuerdo.

Al preguntar "El equipo directivo crea un ambiente de trabajo donde se promueve la integridad (verdad, honestidad, confiabilidad)" el 28\% dice estar totalmente de acuerdo, $39 \%$ de acuerdo, $22 \%$ ni de acuerdo ni en desacuerdo, $9 \%$ en desacuerdo y el $2 \%$ totalmente en desacuerdo. 
En respuestas a "Predomina la rutina, el miedo a la autoridad, la crítica, las sanciones" el 7\% dice estar totalmente de acuerdo, $22 \%$ de acuerdo, $30 \%$ ni de acuerdo ni en desacuerdo, $30 \%$ en desacuerdo y el $11 \%$ totalmente en desacuerdo.

En la pregunta "En la SSNL se estimula a las actividades de convivencia" el 9\% expresó estar totalmente de acuerdo, $46 \%$ de acuerdo, $24 \%$ ni de acuerdo ni en desacuerdo, $17 \%$ en desacuerdo y el $4 \%$ totalmente en desacuerdo.

La figura 5 corresponde a la pregunta "Considero que mi trabajo realmente contribuye al éxito de la SSNL" y el 95\% expresó estar totalmente de acuerdo y de acuerdo, 5\% ni de acuerdo ni en desacuerdo, $0 \%$ en desacuerdo y totalmente en desacuerdo.

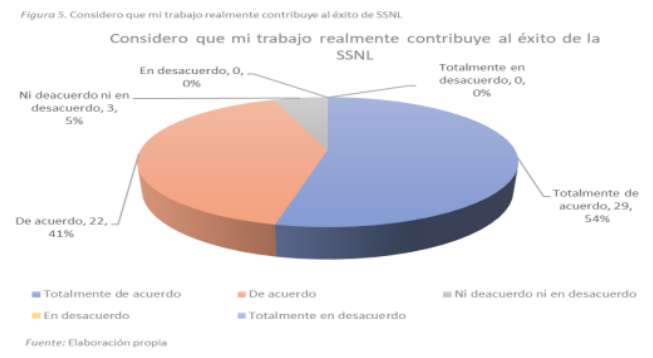

La figura 6 muestra las respuestas de "Estoy comprometido/a con la SSNL" el $70 \%$ dijo estar totalmente de acuerdo, $28 \%$ de acuerdo, $2 \%$ ni de acuerdo ni en desacuerdo, $0 \%$ en desacuerdo y totalmente en desacuerdo.

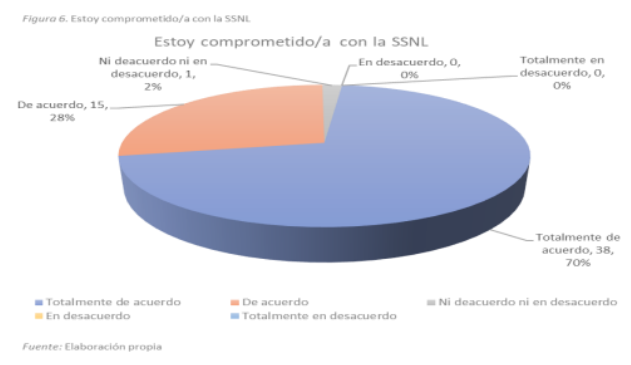

Para el ítem "Recomiendo a la SSNL como un excelente sitio de trabajo" $28 \%$ dice estar totalmente de acuerdo, más del $50 \%$ de acuerdo, y solamente el 19\% suma los rubros de ni de acuerdo ni en desacuerdo, en desacuerdo y totalmente en desacuerdo.

\section{5.- CONCLUSIONES}

$\mathrm{Si}$ las organizaciones se componen de personas, la gestión de estas constituye el elemento básico para cuidar la organización y gestionar el talento humano eficientemente. El liderazgo, la comunicación interna y las relaciones grupales e interpersonales como habilidades gerenciales son elementos de suma importancia para el clima laboral.

La satisfacción laboral de los funcionarios incide sobre el clima organizacional de la Subdirección de Recursos Humanos es decir que en la medida que el clima organizacional se perciba de forma positiva los funcionarios realizarán con mayor satisfacción las funciones cotidianas que favorece el cumplimiento de la misión del Organismo. Lo anterior se evidencia en razón a que el resultado de esta investigación arroja conocimiento sobre la percepción de los responsables de Recursos Humanos del Organismo quienes son los encargados de trasmitir todos los procesos de la Gestión de $\mathrm{RH}$ al resto de los empleados de la salud. "El enfoque subjetivista o perceptual reside en el hecho de que el comportamiento de un trabajador no es una resultante de los factores organizacionales existentes, sino que depende de las percepciones y satisfacción de expectativas que tenga el trabajador". (Noriega, 2011)

La relación entre personas y organizaciones está cambiando a través del tiempo y con rapidez. Stephen Robbins E. (1996) dijo que "No solo hay para enfatizar que las organizaciones tienen diferentes grados de calor humano, sino también reafirmar que cuenta con tradiciones, valores, costumbres, prácticas y procesos de socialización que prevalecen durante el tiempo".

$\mathrm{Si}$ se considera al talento humano como el capital más importante para las organizaciones, debe existir una correcta gestión del mismo. Chiavenato (2017) aseveró que "La gestión de este talento no es una tarea muy sencilla; cada persona es diferente, un fenómeno sujeto a la influencia de muchas variables y entre ellas existen diferencias de aptitudes y patrones de comportamientos que son muy diversos". 
Chiavenato (2011) comenta que "Las tres características principales de las personas mentalmente sanas son: las personas se sienten bien consigo mismas; se sienten bien en relación con otras personas y; son capaces de enfrentar las demandas de la vida y de las circunstancias". (p.74) Revisando estas directrices se puede concluir que el grupo analizado cumple con estas características.

Después de obtener este diagnóstico situacional, es imperante la elaboración de un Plan de Intervención a partir de los resultados obtenidos en esta Investigación, ya que existe especial interés en que el Área de Recursos Humanos logre destacar por su comportamiento y resultados a la organización. La postura es creer firmemente en que los cambios organizacionales pueden darse con la colaboración de todos los integrantes del equipo de trabajo. Se trabajará bajo tres estrategias: primera, el fortalecimiento del liderazgo en los cargos del nivel directivo; segunda, el fortalecimiento de la comunicación interna y tercera el fortalecimiento de los grupos de trabajo. Este Plan de Intervención involucrará, además del Área de Recursos Humanos Central a los responsables de las Áreas de Recursos Humanos de las Unidades Desconcertadas del Organismo, aunque no fueron objeto de la muestra de estudio de investigación, son los símiles de la Central en esas unidades.

En este sentido y de acuerdo al conocimiento de la percepción de los integrantes del equipo de Recursos Humanos, es muy importante involucrar a los que no tiene una buena percepción sobre el clima laboral en esta área, pero es igual de importante mantener a los que si la tienen para que ambos estratos puedan sentirse satisfechos y tengan la posibilidad de desempeñar sus funciones en equilibrio y armonía aún mayor.

Gestionar para provocar un clima laboral agradable es incuestionable dentro de las organizaciones de la Administración Pública y escalarlo al nivel de política pública sería por demás coadyuvante para el logro de la misión, visión, objetivos y metas establecidas por las organizaciones. Un abordaje profesional es importante en este proceso. El área de Recursos Humanos debe dar respuestas efectivas y aportarle al Organismo ventajas competitivas para provocar en el resto de las áreas y los empleados la comunicación correcta para la gestión de sus procesos y aplicando un liderazgo participativo para la correcta diligencia de los mismos, siendo así Modelo a Seguir para todos. 


\section{REFERENCIAS}

Álvarez Torres, S.H., Riaño-Casallas, M. I. (2018). La política pública de seguridad y salud en el trabajo: el caso colombiano. Revista Gerencia y Políticas de Salud, 2018, 17(35), ISSN: 16577027. http://www.redalyc.org/articulo.oa?id=54557477015

Adaptado de HUNT, JAMES. Leadership: A New Synthesis, Thousand Oaks, California, Sage, 1991, p. 221.

Cardona, C. y Trejos, J. (2020). Estudio cualitativo del aprendizaje experiencial para equipos de trabajo organizacional. Revista de Ciencias Sociales (Ve), XXVI (3), 71-82 https://dialnet.unirioja.es/servlet/articulo?codigo $=7565468$

Chiavenato, I. (2009). Comportamiento organizacional. La dinámica del éxito en las organizaciones. Mc Graw Hill.

https://www.gob.mx/cms/uploads/attachment/file/335680/Comportamiento_organizacional._ La_dina_mica_en_las_organizaciones..pdf

Chiavenato, I. (2011). Administración de recursos humanos. El capital humano de las organizaciones. Mc Graw Hill.

http://repositorio.uasb.edu.bo:8080/bitstream/54000/1145/1/Chiavenato-

Recursos\%20humanos\%209na\%20ed.pdf

Chiavenato, Idalberto (2017) Administración de Recursos Humanos. El Capital Humano de las Organizaciones. Mc Graw Hill. P.p (72-73)

Calderón G. Aproximación a un modelo de gestión humana que agregue valor a la empresa colombiana. Universidad Nacional de Colombia: Bogotá; 2008.

Calderón G, Álvarez C. M. Características y sentido de las prácticas de gestión humana en las pequeñas empresas. Revista Universidad Eafit. 2006; 42:26-45.

Gómez Rada CA. (2004). Diseño, construcción y validación de un instrumento que evalúa clima organizacional en empresas colombianas, desde la teoría de respuesta al ITEM. Acta Colombiana de Psicología. (11):97- 113.

https://www.redalyc.org/pdf/798/79801108.pdf

Lugo Garfias, M. E. (2017). Acoso laboral “mobbing”. Comisión Nacional de los Derechos Humanos.

http://appweb.cndh.org.mx/biblioteca/archivos/pdfs/Acoso-Laboral-Mobbing.pdf

LUTHANS, FRED. Organizational Behavior, Nueva York, McGraw-Hill,Higher Education, 2002, p. 123.

Menárguez Puche JF, Saturno Hernández PJ. Descripción del clima organizacional en equipos de atención primaria de una comunidad autónoma. Aten Primaria 1999;23(5):280-4

Salinas-Oviedo C, Laguna-Calderón J, Mendoza-Martínez MR. La satisfacción laboral y su papel en la evaluación de la calidad de la atención médica. Salud Publica Mex 1994;36(1):22-9.

Menárguez Puche JF, Saturno Hernández PJ, López Santiago A. Validación de un cuestionario para la medición del clima organizacional en centros de salud. Aten Primaria 1999;23:198-203.

Noriega Bravo, V. D. L. M., \& Pría Barros, M. D. C. (2011). Instrumento para evaluar el clima organizacional en los Grupos de Control de Vectores. Revista cubana de salud pública, 37(2):116-22 $\quad$ https://www.scielosp.org/pdf/rcsp/2011.v37n2/10.1590/S086434662011000200004/es

ROBBINS Stephen (1996) Comportamiento Organizacional, Teoría y Práctica. 7ma Ed. Prentice Hall. P.p (102) 
Noriega Bravo, V. D. L. M., \& Pría Barros, M. D. C. (2011). Instrumento para evaluar el clima organizacional en los Grupos de Control de Vectores. Revista cubana de salud pública, 37(2):116-22 $\quad$ https://www.scielosp.org/pdf/rcsp/2011.v37n2/10.1590/S086434662011000200004/es

SCHEIN, EDGAR. (1992) Organizational Culture and Leadership, San Francisco, California, JosseyBass, 1992

Ospino Hernández, C. G. (2019). El Rol de las Habilidades Socioemocionales para la Productividad en los Mercados Laborales del Siglo XXI. Nota técnica del BID, 1687. https://fundacionforge.org/archivos/originales/cf0592b46153d2d09627920aa112b675.pdf

Sánchez Torres, R. M. (2011). Política pública laboral del gobierno de Álvaro Uribe 20022010. RELET-Revista Latinoamericana de Estudios del Trabajo, 16(26), 183-218. http://alast.info/relet/index.php/relet/article/view/148 